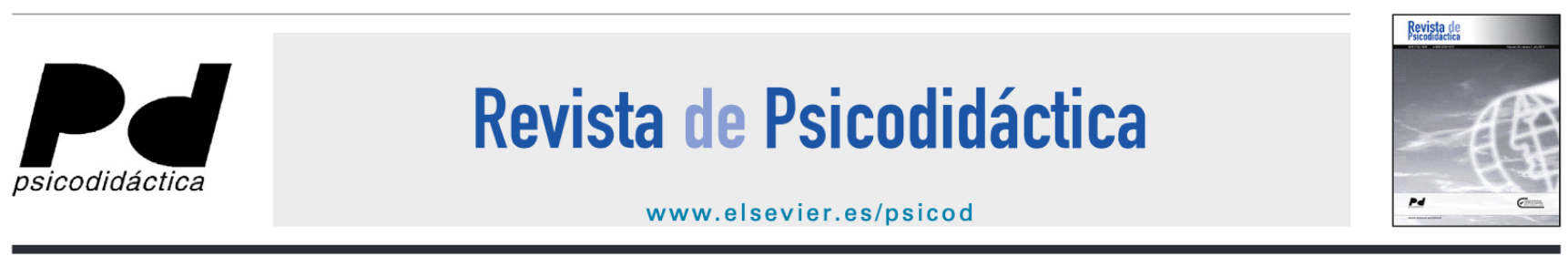

Original

\title{
Modelo de predicción de los estilos de vida saludables a través de la Teoría de la Autodeterminación de estudiantes de Educación Física
}

\author{
Marta Leyton ${ }^{\mathrm{a}}$, Marco Batista ${ }^{\mathrm{b}}$, y Ruth Jiménez-Castuera ${ }^{\mathrm{c}, *}$ \\ ${ }^{a}$ Departamento de Economía Financiera y Contabilidad e Idioma Moderno, Centro de Estudios del Deporte, Universidad Rey Juan Carlos, Madrid, España \\ ${ }^{\mathrm{b}}$ Sport, Health and Exercise Research Unit (SHERU) - Polytechnic Institute of Castelo Branco, Castelo Branco, Portugal \\ c ADICO Deporte (Análisis Didáctico y Comportamental del Deporte), Facultad de Ciencias del Deporte, Universidad de Extremadura, Cáceres, España
}

\section{INFORMACIÓN DEL ARTÍCULO}

\section{Historia del artículo:}

Recibido el 26 de enero de 2019

Aceptado el 29 de mayo de 2019

On-line el 11 de noviembre de 2019

\section{Palabras clave:}

Motivación

Necesidades psicológicas básicas

Hábitos alimenticios

Educación Física

Estudio instrumental

\begin{abstract}
R E S U M E N
El objetivo principal de este estudio es analizar la predicción que los procesos motivacionales tienen en la adopción de hábitos de estilos de vida que realzan la salud en adolescentes, en las clases de Educación Física. La muestra se compone por 214 estudiantes de Enseñanza Secundaria en Portugal, entre los 15 y 19 años $(M=16.46, D T=.96)$, de ambos sexos (104 chicos y 110 chicas). Se administran los siguientes cuestionarios: Escala del Locus Percibido de Causalidad en Educación Física, Escala de medición de las necesidades psicológicas básicas, el Cuestionario de Estilos de Vida Saludables y la Escala de Medida de la Intencionalidad para ser Físicamente Activo. Se utiliza un análisis de ecuaciones estructurales, y se muestra que la satisfacción de las necesidades psicológicas básicas predice la motivación intrínseca, y esta a su vez predice las variables relacionadas con los estilos de vida que realzan la salud, ambas predicciones de forma positiva y significativa. Se destaca la relevancia de desarrollar la motivación más autodeterminada a través de la satisfacción de las necesidades psicológicas básicas en las clases de Educación Física para fomentar estilos de vida saludables en el alumnado.
\end{abstract}

(ㅇ 2019 Universidad de País Vasco. Publicado por Elsevier España, S.L.U. Todos los derechos reservados.

\section{Prediction model of healthy lifestyles through the self-determination theory in physical education students}

\begin{abstract}
A B S T R A C T
The main objective of this study was to analyze the prediction that motivational processes have in the adoption of lifestyle habits that enhance adolescent health in Physical Education classes. The sample consisted of 214 Secondary Education students in Portugal, between 15 and 19 years old ( $M=16.46$, $S D=.96$ ), of both genders ( 104 boys and 110 girls). The following questionnaires were administered: Scale of the Locus Perceived of Causality in Physical Education, Scale of measurement of the basic psychological needs, the Questionnaire of Healthy Lifestyles and the Scale of Measurement of Intentionality to be Physically Active. An analysis of structural equations was used, which showed that the satisfaction of basic psychological needs predicted the intrinsic motivation, and this in turn, predicted the variables related to healthy lifestyles, both positively and significantly. The importance of developing self-determined motivation through the satisfaction of basic psychological needs in Physical Education classes to promote healthy lifestyles in our students, is highlighted.
\end{abstract}

๑ 2019 Universidad de País Vasco. Published by Elsevier España, S.L.U. All rights reserved.

\footnotetext{
* Autora para correspondencia.

Correo electrónico: ruthji@unex.es (R. Jiménez-Castuera).
} 


\section{Introducción}

La promoción de estilos de vida saludables, particularmente los referidos a la práctica de actividad física (AF) y al desarrollo de adecuados hábitos alimenticios, constituye una de las labores primordiales de los profesionales de la Educación Física (EF) (Usán, Salavera, Mejías, y Murillo, 2018).

La EF es un escenario donde los jóvenes realizan ejercicio físico, siendo un medio para fomentar la motivación y la adherencia para realizar una práctica deportiva en horario extraescolar (Franco, Coterón, Gómez, Brito, y Martínez 2017). El docente de EF es uno de los principales promotores de la práctica de $\mathrm{AF}$ (Egan y Webster, 2018) y su figura es crucial para que el alumnado aumente o no la práctica de AF (Yilmaz, Esenturk, Demir, e Ilhan, 2017). Thompson et al. (2014) determinan que el profesorado es el motor de la motivación del alumnado hacia la $\mathrm{AF}$, a fin de que se mantenga activo para el logro de hábitos saludables, señalando la importancia concedida a la motivación como factor favorecedor de un estilo de vida activo y saludable.

Una de las teorías que ayuda a explicar la motivación de los estudiantes en las clases de EF es la Teoría de la Autodeterminación (TAD) (Deci y Ryan, 1985; Ryan y Deci, 2000). La TAD propone que la motivación se encuentra enmarcada a lo largo de un continuo en el que se distinguen tres niveles (Vansteenkiste, Lens, y Deci, 2006; Vansteenkiste, Niemiec, y Soenens, 2010): motivación intrínseca (la más autodeterminada, realizar una actividad por propio placer), motivación extrínseca (realizar una actividad por recompensa o reconocimientos externos) y desmotivación (la menos autodeterminada) (Deci y Ryan, 2000).

Asimismo, establece tres necesidades psicológicas básicas (NPB): autonomía (comprometerse en actividades por propia elección), competencia (interactuar de manera eficiente con el medio para sentirse competente) y relaciones sociales (sentirse parte de un grupo) (Deci y Ryan, 2000; Ryan y Deci, 2000). En la TAD, las NPB constituyen los mediadores psicológicos que influirán en los tres principales tipos de motivación (Deci y Ryan, 1985; Ryan y Deci, 2000). Numerosos estudios utilizan las NPB como mediadores que predicen positivamente las formas de motivación más autodeterminadas (González-Cutre, Sicilia, y Moreno, 2011; McDonough y Crocker, 2007; Standage, Duda, y Ntoumanis, 2006).

El Modelo Jerárquico de la Motivación (MJM) (Vallerand, 1997, 2001) asocia las NPB con la TAD (Deci y Ryan, 2002). El modelo establece que los aspectos sociales del entorno (variables antecedentes) influyen en la motivación en función de la consecución o no de una serie de NPB (autonomía, competencia y relaciones sociales), cuya satisfacción incrementa el grado de motivación intrínseca (formas de motivación autodeterminada) (Deci y Ryan, 2000; Ryan y Deci, 2000) y dará lugar a consecuencias positivas a nivel cognitivo, afectivo y conductual (variables consecuentes), las cuales corresponden a las variables de estilos de vida saludables analizadas.

Está demostrado que el alumnado que muestra una mayor satisfacción de las NPB desarrollan una motivación más autodeterminada (González-Cutre et al., 2014). Los procesos motivacionales desarrollados por el alumnado en el contexto de la EF actúan como elementos determinantes en los comportamientos desarrollados durante las clases (Charchaoui, Cachón, Chacón, y Castro, 2017). El estudio de los procesos motivacionales en EF es de gran interés dado que la motivación ha sido entendida como factor determinante para el mantenimiento de conductas que realzan salud (Ntoumanis, Quested, Reeve, y Cheon, 2017).

Sin embargo, ¿cómo se considera a un estilo de vida saludable? Existe un consenso universal que implica que todo lo relacionado con la nutrición adecuada, la práctica de AF, buenos hábitos de descanso y la higiene personal fomenta conductas saludables; por otro lado, el estrés, la falta de descanso, el tabaquismo, un estilo de vida sedentario y el abuso de alcohol y/o sustancias psicoactivas constituyen un riesgo para la salud (Casado-Pérez et al., 2015).

Fernández-Ozcorta, Almagro, y Sáenz-López (2015) afirman que aquel alumnado que tenga satisfechas sus NPB y una motivación más autodeterminada tendrán una mayor intención de seguir siendo físicamente activos en el futuro. En esta línea, Franco, Coterón, Gómez, y Laura (2017), confirman la predicción de la motivación intrínseca sobre la intención de realizar práctica de AF en el futuro.

Resultados de diversas investigaciones determinan que la utilización de estrategias por parte del docente, de aspectos psicológicos positivos, como el aumento de la motivación intrínseca del alumnado en las clases de EF, posibilitan el desarrollo y consolidación de conductas saludables relacionadas con la AF (Aspano, Lobato, Leyton, Batista, y Jiménez, 2016; Sánchez-Oliva, PulidoGonzález, Leo, González-Ponce, y García-Calvo, 2017; Standage, Duda, y Ntoumanis, 2005; Taylor, Ntoumanis, Standage, y Spray, 2010), y adecuados hábitos alimenticios y de descanso (Giménez, López, Urda, y Collazos, 2015; Jiménez, Cervelló, García, SantosRosa, e Iglesias, 2007).

Por lo tanto, considerando la importancia que supone la motivación del alumnado en las clases de EF para el desarrollo de hábitos saludables, el objetivo principal de este estudio es analizar la predicción que los procesos motivacionales tienen en la adopción de hábitos de estilos de vida que realzan la salud en las clases de EF. Se establece la hipótesis de que la satisfacción de las NPB por parte del alumnado predecirá de forma positiva la motivación intrínseca, y esta a su vez predecirá las variables relacionadas con los estilos de vida que realzan la salud (alimentación equilibrada, respeto al horario de las comidas, adecuados hábitos de descanso e intención de ser físicamente activo).

\section{Método}

\section{Diseño de investigación}

El estudio se ubica dentro de los estudios empíricos cuantitativos y, dentro de estos, hace referencia a un estudio descriptivo de poblaciones mediante encuestas (Montero y León, 2007).

\section{Participantes}

Los participantes pertenecen a una población de estudiantes de los tres últimos años de Enseñanza Secundaria de diferentes centros educativos públicos de Setúbal, Sesimbra y Lisboa, en Portugal. Son 214 sujetos de ambos sexos (104 chicos y 110 chicas) cuyas edades oscilan entre los 15 y 19 años $(M=16.46, D T=.96)$. Ochenta y tres 83 discentes eran de $7 .^{\circ}$ año, 23 de $8 .^{\circ}$ año, y 105 de $9 .^{\circ}$ año. Para la selección de los participantes se utiliza un muestreo por conglomerados elegidos de forma intencionada (Azorín y SánchezCrespo, 1986). Cada conglomerado está constituido por un aula de unos 19-23 discentes, obteniendo 10 conglomerados.

\section{Instrumentos}

A continuación se presentan las variables utilizadas en el estudio junto al instrumento de medida utilizados para su análisis.

Nivel de motivación: Para medir el nivel de motivación del alumnado en las clases de EF, se utiliza la Escala del Locus Percibido de Causalidad en Educación Física (Perceived Locus of Causality Scale, PLOC Scale). Se utiliza la validación portuguesa (Fernandes y Vasconcelos-Raposo, 2005) de la PLOC Scale de Goudas, Biddle, y Fox (1994). Consta de 20 ítems, divididos en cinco factores. En el presente estudio se utiliza el factor motivación intrínseca, compuesto por cinco ítems. Los índices de bondad de ajuste para la presente escala, tras el análisis factorial confirmatorio (AFC), son 
aceptables (Hu y Bentler, 1999): chi cuadrado $\left(\chi^{2}\right)=142.55, p=.00$ chi cuadrado divido por los grados de libertad $\left(\chi^{2} \mid g l\right)=2.04$, índice CFI (Comparative Fit Index, CFI) $=.97$, índice de Bollen's $(\mathrm{IFI})=.97$, Goodness Fit Index $(\mathrm{GFI})=.92$, Standarized RMR $(\mathrm{SRMR})=.03$, Root Mean Square Error of Approximation (RMSEA) $=.07$.

Satisfacción de las NPB: Se utiliza la Escala de medición de las necesidades psicológicas básicas (Vlachopoulos y Michailidou, 2006), adaptada y validada al portugués por Pires, Cid, Borrego, Alves, y Silva (2010). Consta de 12 ítems, divididos en tres factores: autonomía, competencia y relaciones sociales. Los índices de bondad de ajuste en el AFC son aceptables (Hu y Bentler 1999): $\chi^{2}=111.59, p=.00, \chi^{2} / g l=2.07, \mathrm{CFI}=.97, \mathrm{IFI}=.97, \mathrm{GFI}=.92$ $\mathrm{SRMR}=.06, \mathrm{RMSEA}=.07$.

Estilos de vida saludable: Para determinar el estilo de vida se utiliza el Cuestionario de Estilos de Vida Saludables, diseñado por Wold (1995), traducido y validado al portugués por Batista, Jiménez, Leyton, Lobato, y Aspano (2016). Consta de 12 ítems divididos en cuatro factores, de los cuales se utilizan: alimentación equilibrada, respeto al horario de las comidas y hábitos de descanso. Los índices de bondad de ajuste del AFC son aceptables (Hu y Bentler, 1999): $\chi^{2}=632.68, p=.00, \chi^{2} \mid g l=2.01, \mathrm{CFI}=.94, \mathrm{IFI}=.94, \mathrm{GFI}=.92$, $\mathrm{SRMR}=.06, \mathrm{RMSEA}=.07$.

Intención de ser fisicamente activo: Se utiliza la Escala de Medida de la Intencionalidad para ser Físicamente Activo (MIFA) creada por Hein, Müür, y Koka (2004), traducida y validada al portugués por Jiménez, Leyton, y Batista (2019). Esta escala consta de cinco ítems que se agrupan en un único factor. Tras el AFC, los índices de bondad de ajuste del constructo son aceptables (Hu y Bentler, 1999): $\chi^{2}=18.81, p=.00, \chi^{2} / g l=3.23, \mathrm{CFI}=.97, \mathrm{IFI}=.97, \mathrm{GFI}=.97$, $\mathrm{SRMR}=.03, \mathrm{RMSEA}=.06$.

En todos los cuestionarios utilizados, todos los ítems se responden a través de una escala tipo Likert de 5 puntos, cuyo rango va desde el valor 0 , totalmente en desacuerdo a 5 , totalmente de acuerdo a la formulación de la pregunta.

\section{Procedimiento}

El estudio se aprueba por el Comité Ético de la Universidad de Extremadura. Para la recogida de información se contacta con los centros de enseñanza secundaria. Se informa de los objetivos del estudio y se pide el pertinente permiso para que el alumnado participe en la investigación. Se pide un consentimiento informado de los progenitores/tutores ya que la mayoría del alumnado son menores de edad. Se realiza la administración de los cuestionarios, sin estar presente el profesorado en la clase y bajo la supervisión del investigador, se insiste en el anonimato de las repuestas y en que se contesten con sinceridad. Se administran los cuestionarios en el segundo semestre, en el horario lectivo. El tiempo requerido para cumplimentar los cuestionarios es de 20 minutos, aproximadamente.

\section{Análisis de los datos}

En primer lugar se constata la existencia de normalidad en los datos obtenidos. Para los análisis de normalidad univariada se utiliza, primero, los indicadores de asimetría y curtosis de las variables. Curran, West, y Finch (1996) establecen los límites de asimetría y curtosis en valores absolutos. Valores de, hasta 2 para la asimetría y 7 para la curtosis, son considerados normales; valores comprendidos entre 2 y 3 para la asimetría y entre 7 y 21 para la curtosis, son considerados moderadamente normal; $y$ valores superiores a 7 en la asimetría y a 21 en la curtosis, son considerados no normal. Posteriormente, para comprobar la normalidad multivariada se realiza el test de Mardia (Mardia, 1970), el cual, de acuerdo con Bollen
(1989) debe ser inferior a $p(p+2)$, siendo $p$ el número de variables observadas.

Posteriormente se realiza el AFC con el objetivo de evaluar la validez de constructo, respetando el criterio de eliminar aquellos ítems cuyo peso de regresión no presentan un valor adecuado (superior a .40) (Revelle, 2014). Además, se halla el modelo de medición para comprobar la validez de los factores que se incluyen en el Modelo de Ecuaciones Estructurales (SEM). La matriz de covarianzas es utilizada como input para el análisis de los datos.

A continuación, se realiza un análisis de fiabilidad, para comprobar la consistencia interna de los cuestionarios. Para el análisis de fiabilidad, se utiliza el alfa de Cronbach, igual o mayor que .70 (Nunnally, 1978), así como el coeficiente omega (McDonald, 1999), que también sirve para verificar la consistencia interna de las variables utilizadas en la investigación y, según algunos autores (Revelle y Zinbarg, 2009), demuestra evidencia de mayor exactitud. En el coeficiente omega de McDonald, el rango establecido está entre 0 y 1 , proporcionando los valores más altos las mediciones más fiables (Revelle y Zinbarg, 2009). Sin embargo, según Campo-Arias y Oviedo (2008), para considerar un valor aceptable de confiabilidad mediante el coeficiente omega, estos deben ser superiores a .70 .

Además, se estima la fiabilidad compuesta (grado de consistencia entre los indicadores con el constructo latente) y la varianza media extractada (la cantidad de la varianza de los indicadores capturada por el constructo comparada con la capturada por el error de medida). En cuanto a la fiabilidad compuesta el nivel mínimo es de .70, y la varianza media extractada debe ser mayor de .50, para concluir que una cantidad sustancial de la varianza es capturada por el constructo (Arias, 2008).

Resaltar que se crean las variables correspondientes con los ítems que muestran adecuada validez y fiabilidad, y se calculan los estadísticos descriptivos. Posteriormente se plantea el SEM, ya que es considerada la herramienta más potente para el estudio de las relaciones de predicción entre las variables (Aron y Aron, 2001). Este análisis se lleva a cabo utilizando el método de estimación máxima verosimilitud (maximum likelihood, ML). La ventaja del SEM es que permite tratar modelos teóricos incluyendo todas las variables dentro de una misma ecuación de regresión. A su vez, este cálculo de ecuaciones estructurales muestra una serie de coeficientes (índices fit o de bondad de ajuste) que permiten comprobar la bondad o semejanza del modelo teórico propuesto con los datos empíricos. Entre ellos se analiza la significatividad de $\chi^{2}\left(p\right.$ de $\left.\chi^{2}\right)$, que indica el parecido entre las covarianzas observadas con aquellas que se encuentran en el modelo hipotético; valores no significativos en este índice indican una correspondencia aceptable entre el modelo propuesto y los datos. El $\chi^{2} / g l$ constituye un índice menos sensible al tamaño de la muestra que el índice fit anterior, de modo que valores por debajo de 2 son considerados como indicadores de muy buen ajuste del modelo, mientras que valores por debajo de 5 son considerados como aceptables (Hu y Bentler, 1999). El índice CFI y el GFI toman como valores entre 0 y 1 ; valores superiores a .90 son considerados como aceptables (Bentler, 1995; Byrne, 2010). En el IFI, que toma valores entre 0 y 1 , se considera que el modelo se ajusta a los datos empíricos cuando alcanza valores superiores a .90. Otro índice fit es el RMSEA, cuyos valores inferiores a .10 son considerados como aceptables (Byrne, 2010). Por último, se contempla el grupo de índices fit con el SRMR, de modo que valores por debajo de .08 se consideran como aceptables (Hu y Bentler, 1999). Además, como indican Hu y Bentler (1999), es recomendable la contemplación de varios de estos índices para aceptar o rechazar un modelo, no pudiéndose aceptar con uno solo de estos índices o por el contrario rechazarlo por el incumplimiento de uno solo de los índices de ajustes.

Se estiman los efectos indirectos en el SEM: el efecto de las NPB de autonomía, competencia y relaciones sociales sobre la alimentación equilibrada, el respeto al horario de las comidas, los hábitos 
Tabla 1

Asimetría y curtosis de las variables objeto de estudio

\begin{tabular}{lll}
\hline Instrumento / Variable & Asimetría & Curtosis \\
\hline Motivación intrínseca & -.76 & .32 \\
NPB autonomía & -.34 & -.24 \\
NPB competencia & -.56 & .12 \\
NPB relaciones sociales & -1.06 & .81 \\
Alimentación equilibrada & -.51 & -.35 \\
Respeto horario comidas & -.70 & -.24 \\
Hábitos descanso & -.46 & -.52 \\
Intención de ser físicamente activo & -.70 & -.42 \\
\hline
\end{tabular}

Tabla 2

Estadísticos descriptivos y análisis de fiabilidad

\begin{tabular}{lllllll}
\hline Variable & $M$ & $D T$ & $\alpha$ & $\omega$ & FC & VME \\
\hline Motivación intrínseca & 3.86 & .91 & .85 & .95 & .80 & .81 \\
NPB autonomía & 3.31 & .85 & .73 & .85 & .80 & .71 \\
NPB competencia & 3.92 & .76 & .77 & .91 & .81 & .78 \\
NPB relaciones sociales & 4.36 & .70 & .81 & .93 & .81 & .60 \\
Alimentación equilibrada & 3.66 & .90 & .78 & .84 & .80 & .61 \\
Respeto horario comidas & 3.78 & .95 & .76 & .82 & .83 & .52 \\
Hábitos descanso & 3.59 & .99 & .66 & .72 & .74 & .51 \\
Intención de ser físicamente activo & 3.92 & .91 & .81 & .90 & .82 & .61 \\
\hline
\end{tabular}

DT: desviación típica, M: media, FC: fiabilidad compuesta, NPB: necesidad psicológica básica, VME: varianza media extractada, $\alpha$ : alfa de Cronbach, $\omega$ : coeficiente omega.

de descanso y la intención de ser físicamente activo, a través de la motivación intrínseca. Los valores $p<.05$ y $p<.01$ son utilizados para la significación estadística.

Para el análisis de normalidad univariada (asimetría y curtosis), fiabilidad (alfa de Cronbach) y descriptivos se usa el programa estadístico SPSS 21.0. Los análisis de normalidad multivariada (coeficiente Mardia), AFC, modelo de medición y SEM, se llevan a cabo con el programa E.Q.S. 6.1.

Respecto al índice omega de McDonald, los cálculos se realizan con el "psych» 1.4.2.3 (Revelle, 2014) de R 3.0.3 (R Core-Team, 2014).

\section{Resultados}

\section{Análisis de normalidad}

Las medidas de asimetría y curtosis verifican la normalidad univariada (ver Tabla 1). El coeficiente de Mardia arroja un valor (147.01) inferior a " $p(p+2)$ », por lo que siguiendo a Bollen (1989), se considera que existe normalidad multivariada.

\section{Estadísticos descriptivos y análisis de fiabilidad de los} instrumentos de medida

En la Tabla 2 se observan los estadísticos descriptivos de los instrumentos empleados en el estudio. La tabla recoge la $M$ y la $D T$ de todas las variables del estudio, se observa que la NPB de relaciones sociales obtiene la media más alta, en contraposición con la NPB de autonomía que obtiene la media más baja.

También se observan los resultados del análisis de fiabilidad, en la que todos los factores presentan una adecuada fiabilidad (alfa de Cronbach >.70) (Nunnally, 1978). Sin embargo, tal y como se observa en la Tabla 2, el factor hábitos de descanso muestra una fiabilidad inferior a la recomendada (.66), pero dado el pequeño número de ítems que lo componen (tres), la consistencia interna observada puede ser marginalmente aceptada (Hair, Anderson, Tatham, y Black, 1998; Nunnally y Bernstein, 1994). Con relación al coeficiente omega los resultados presentan valores por encima de .70 (Campo-Arias y Oviedo, 2008).
En la Tabla 2 se observa la fiabilidad compuesta y la varianza media extractada, se obtienen valores por encima de .70 para la fiabilidad compuesta y mayores de .50 para la varianza media extractada (Arias, 2008).

\section{Modelo de medición}

Se realiza un AFC con el objetivo de evaluar la validez de constructo y seleccionar las variables que se incluyen en los análisis SEM, realizando así el modelo de medición, tal y como pueden observarse en la Figura 1. Destacar que el modelo muestra adecuados índices de ajuste $\left(p=.00, \chi^{2} / g l=2.01, \mathrm{CFI}=.95, \mathrm{IFI}=.95, \mathrm{GFI}=.92 ; \mathrm{SRMR}=.05\right.$, RMSEA = .05) (Hu y Bentler, 1999; Kline, 2011).

\section{Modelo de ecuaciones estructurales}

En línea con el MJM (Vallerand, 1997; Vallerand, 2001), se incluyen las variables antecedentes (NPB), los mediadores (motivación intrínseca), y las consecuencias (variables del estilo de vida saludable) (ver Figura 2).

En este modelo se pretende conocer las variables predictoras de un estilo de vida saludable, a partir de las NPB y la motivación intrínseca.

En el modelo de ecuaciones estructurales propuesto, la satisfacción de las NPB predicen la motivación intrínseca, y esta a su vez predice los factores relacionados con los hábitos alimenticios (alimentación equilibrada y horarios de comida estables), los hábitos de descanso y la intención de ser físicamente activo. La satisfacción de las NPB de autonomía, competencia y relaciones sociales predicen de forma positiva y significativa la motivación intrínseca ( $\beta=.17 ; \beta=.80 ; \beta=.20$ respectivamente). Y la motivación más autodeterminada predice positiva y significativamente la alimentación equilibrada $(\beta=.50)$, con el factor respeto al horario de las comida $(\beta=.49)$, con los hábitos de descanso $(\beta=.50)$ y con la intención de ser fisicamente activo $(\beta=.95)$.

Indicar también, que los resultados muestran un adecuado ajuste del modelo $\left(p=.00, \chi^{2} / g l=.88, \mathrm{CFI}=.92\right.$, IFI $=.92$, GFI $=.92$, SRMR $=.07$, RMSEA = .06) (Hu y Bentler, 1999; Kline, 2011).

\section{Efectos indirectos entre las variables latentes}

Los efectos indirectos entre las variables latentes se muestran en la Tabla 3.

\section{Discusión}

El presente trabajo tiene como objetivo principal analizar la predicción que los procesos motivacionales tienen en la adopción de hábitos de estilos de vida que realzan la salud en las clases de EF. En la hipótesis se plantea que la satisfacción de las NPB predecirá de forma positiva la motivación intrínseca, y esta a su vez, predecirá variables relacionadas con los hábitos de estilo de vida que realzan la salud. Los resultados obtenidos en el análisis de ecuaciones estructurales confirman la hipótesis planteada.

Estos resultados van en la línea de los encontrados por Menéndez y Fernández-Río (2017) y Núñez y León (2016), quienes señalan que el alumnado que mostraba una mayor satisfacción de las NPB desarrollaba una motivación más autodeterminada hacia la práctica. Estudios previos (Sánchez-Oliva, Leo, Sánchez-Miguel, Amado, y García-Calvo, 2013) evidencian que al alumnado que presentan una motivación más autodeterminada son aquellos que tienen una predisposición más positiva hacia la práctica deportiva.

Se ha demostrado que un mayor sentimiento de autonomía va a hacer que la motivación intrínseca sea más elevada (Amado et al., 2014; Sánchez-Oliva et al., 2017; Standage et al., 2005; Taylor y 


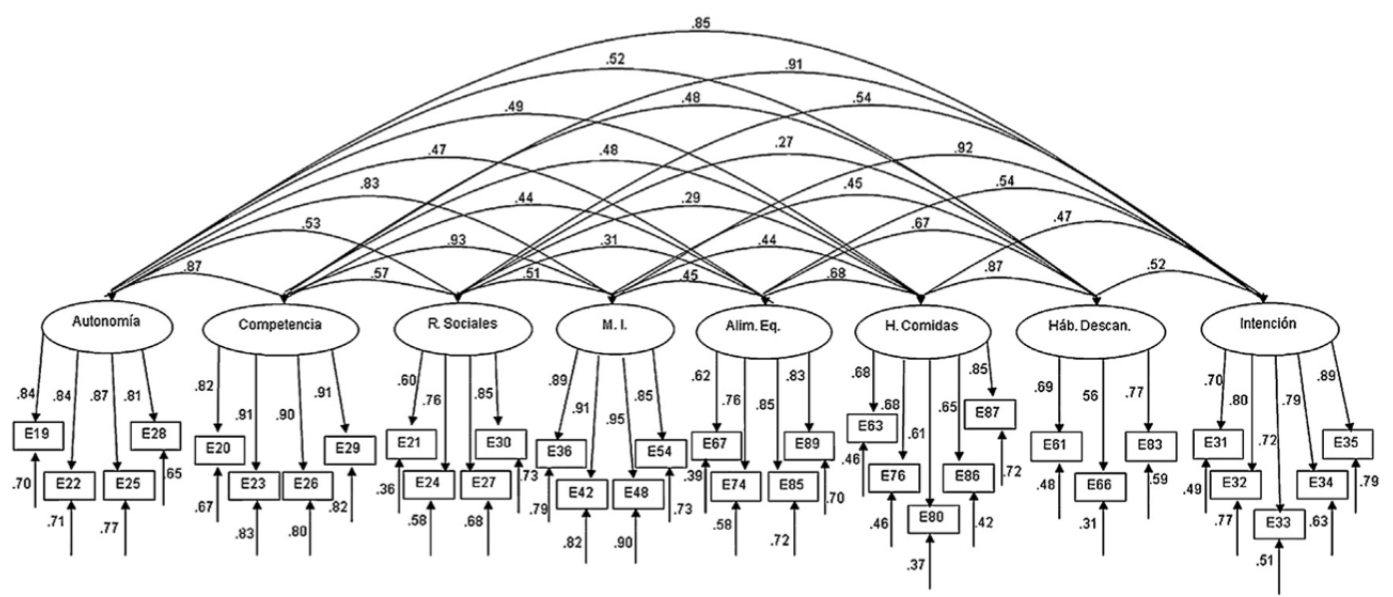

Figura 1. Modelo de medida. Las elipses representan las variables latentes y los rectángulos las variables medidas. Las varianzas explicadas se muestran sobre las flechas pequeñas. Todos los pesos de regresión están estandarizados y son estadísticamente significativos. Alim. Eq.: alimentación equilibrada, H. Comidas: respeto al horario de comidas, Háb. Descan.: hábitos de descanso, Intención: intención de ser físicamente activo, M. I.: motivación intrínseca, R. Sociales: relaciones sociales.

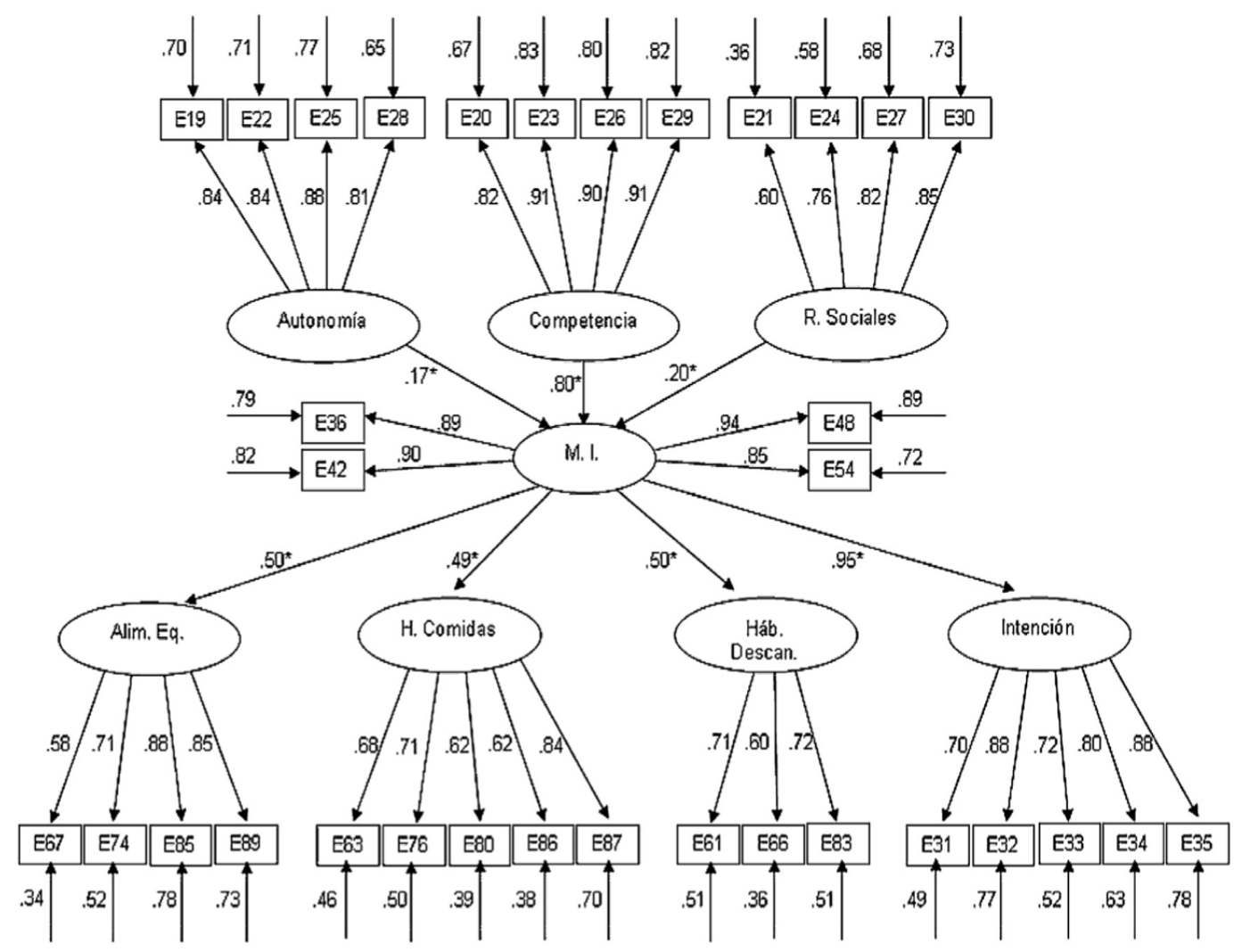

Figura 2. Modelo de ecuaciones estructurales; se muestran las relaciones entre la satisfacción de las necesidades psicológicas básicas de autonomía, competencia y relaciones sociales, la motivación intrínseca y las variables relacionadas con los estilos de vida que realzan la salud. Todos los parámetros están estandarizados y son estadísticamente significativos. Alim. Eq.: alimentación equilibrada, H. Comidas: respeto al horario de comidas, Háb. Descan.: hábitos de descanso, Intención: intención de ser físicamente activo, M. I.: motivación intrínseca, R. Sociales: relaciones sociales.

Ntoumanis, 2007), siendo la NPB que más predice la motivación más autodeterminada. Sin embargo, en el presente estudio, la NPB de competencia es el predictor más fuerte de la motivación intrínseca.

Como se ha demostrado en trabajos recientes (Deci, Olafsen, y Ryan, 2017; Ryan y Deci, 2017), junto con la NPB de autonomía, la competencia explica la variación incremental en la motivación, el bienestar y el desempeño hacia una actividad.
En el modelo propuesto también se observa que la motivación más autodeterminada predijo positivamente las variables de estilos de vida que realzan la salud como son la alimentación equilibrada, el respeto al horario de las comidas, los adecuados hábitos de descanso y la intención de ser físicamente activo. $\mathrm{Ng}$ et al. (2012), en un metaanálisis con la mayoría de los estudios no experimentales, determinan que existe una relación fuerte entre la TAD y conductas positivas de salud. Insisten en la importancia 
Tabla 3

Efectos indirectos del modelo de ecuaciones estructurales

\begin{tabular}{|c|c|c|c|}
\hline & Variables & Beta estandarizado & Valor $t$ \\
\hline \multirow[t]{4}{*}{ NPB autonomía } & $\begin{array}{l}\text { Alimentación } \\
\text { equilibrada }\end{array}$ & $.17^{*}$ & 2.26 \\
\hline & $\begin{array}{l}\text { Respeto al horario de } \\
\text { las comidas }\end{array}$ & -.02 & -.26 \\
\hline & Hábitos de descanso & -.00 & -.09 \\
\hline & $\begin{array}{l}\text { Intención de ser } \\
\text { físicamente activo }\end{array}$ & $.32^{* *}$ & 4.39 \\
\hline \multirow[t]{4}{*}{ NPB competencia } & $\begin{array}{l}\text { Alimentación } \\
\text { equilibrada }\end{array}$ & .09 & 1.14 \\
\hline & $\begin{array}{l}\text { Respeto al horario de } \\
\text { las comidas }\end{array}$ & -.00 & -.09 \\
\hline & Hábitos de descanso & -06 & .70 \\
\hline & $\begin{array}{l}\text { Intención de ser } \\
\text { físicamente activo }\end{array}$ & $.49^{*}$ & 7.44 \\
\hline \multirow[t]{4}{*}{ NPB relaciones sociales } & $\begin{array}{l}\text { Alimentación } \\
\text { equilibrada }\end{array}$ & .15 & 1.90 \\
\hline & $\begin{array}{l}\text { Respeto al horario de } \\
\text { las comidas }\end{array}$ & $.17^{*}$ & 2.08 \\
\hline & Hábitos de descanso & .09 & .94 \\
\hline & $\begin{array}{l}\text { Intención de ser } \\
\text { físicamente activo }\end{array}$ & $.75^{* *}$ & 17.48 \\
\hline
\end{tabular}

de concretar intervenciones basadas en la TAD. En el estudio de Carbó-Carreté, Guàrdia-Olmos, Giné, y Schalock (2016), se determina que los apoyos individualizados en el campo de la AF actúan como predictores de la mejora de la calidad de vida.

Estudios recientes como el de Chacón-Cuberos et al. (2018b) y el de Leyton, García, Fuentes, y Jiménez (2018) apoyan los resultados del presente estudio, encontrando relación entre la motivación hacia la AFy los hábitos alimenticios. Sin embargo, Ferriz, GonzálezCutre, Sicilia, y Hagger (2016), contradicen los resultados obtenidos en el presente estudio, ya que no encuentran ninguna relación entre la motivación en la EF y la alimentación saludable, después de la aplicación de un programa basado en la TAD en clases de EF. Al igual que Chacón-Cuberos, Zurita-Ortega, Olmedo-Moreno, Padial-Ruz, y Castro-Sánchez (2018a), que tampoco encuentran esta relación en una muestra de estudiantes universitarios de EF.

No son muchos los estudios que relacionan la motivación hacia la AF con los hábitos de descanso. Jiménez et al. (2007) encuentran relaciones, aunque no significativas, entre la motivación intrínseca en el alumnado de EF con los hábitos de descanso. Sin embargo, en el presente estudio sí se encuentra una predicción significativa entre ambas variables. Al igual que en el estudio experimental con adultos y mayores, de Leyton, Batista, Lobato, Aspano, y Jiménez (2017), donde señalan que la motivación intrínseca es crucial para el desarrollo de hábitos saludables como son los adecuados hábitos alimenticios y de descanso, de mujeres adultas y mayores.

Con relación a los resultados que determinan que la motivación intrínseca predijo la intención de ser físicamente activo, Sanchez, Byra, y Wallhead (2012) afirman que la motivación supone un conjunto de fenómenos emocionales, cognitivos y sociales, y que, si se utiliza un estilo de enseñanza donde se permite que el alumnado participe en el proceso de enseñanza-aprendizaje, la implicación cognitiva y física será mayor. Trabajos como el de Moreno-Murcia, Huéscar, y Cervelló (2012) corroboran los resultados del presente estudio. Muestran que la motivación intrínseca, predice positivamente la importancia que los estudiantes atribuyen a la EF, y esto, a su vez, predice positivamente la intención del estudiante de continuar haciendo deporte. En la misma línea, Fernández-Ozcorta et al. (2015) afirman que la motivación autodeterminada predice positivamente la intención de ser físicamente activo en adolescentes.
Parece evidente que el ejercicio físico a largo plazo parece ideal para generar una mayor motivación intrínseca $\mathrm{y}$, por lo tanto, modificaciones en los hábitos de vida saludables que son estables a lo largo del tiempo (Saavedra, García-Hermoso, Escalante, y Domínguez, 2014).

Aunque el presente estudio es un avance para conocer el papel que tiene la $\mathrm{EF}$ en la adopción de hábitos relacionados con la salud, es importante realizar un estudio longitudinal y de intervención, para poder establecer estrategias adecuadas para aumentar la satisfacción de las NPB y por lo tanto de la motivación intrínseca. También es interesante realizar una triangulación con datos obtenidos no solo por parte del alumnado, sino por el entorno más cercano a estos, como son el profesorado y/o progenitores, así como ampliar la muestra a otras zonas geográficas y a diferentes tipos de centros.

Ante los resultados obtenidos, estos hallazgos tienen gran aplicación a la hora de plantear tareas por parte del profesorado de $\mathrm{EF}$, fomentar las NPB de autonomía, competencia y relaciones sociales del alumnado, utilizando estrategias como darles la posibilidad de elegir actividades, proponer actividades con diferentes niveles de dificultad, realizar juegos cooperativos y de colaboración para fomentar el trabajo en equipo, entre otras.

Se consideran muy importantes los resultados hallados, ya que tienen fuertes implicaciones en la enseñanza de la EF. El profesorado debe atender a las estrategias para la mejora de las NPB durante las actividades realizadas en clase, de manera específica introduciéndolas en el proyecto docente y en cada unidad didáctica, como competencias a alcanzar por parte del alumnado. Pues esto permitirá que el discente aumente la forma de motivación más autodeterminada, lo que conduce a un incremento de la práctica de AF y un estilo de vida más saludable.

Por lo tanto, se concluye que la satisfacción de las NPB de autonomía, competencia y relaciones sociales, sobre todo la NPB de competencia, predice positiva y significativamente la motivación intrínseca. Y la motivación intrínseca a su vez predice de forma positiva y significativa el mantenimiento de una alimentación equilibrada, el respeto al horario de las comidas, unos adecuados hábitos de descanso y, con un peso de predicción mucho mayor, la intención de ser físicamente activo en un futuro.

\section{AGRADECIMIENTOS}

Este estudio se ha realizado gracias a la aportación de la Consejería de Economía e Infraestructuras de la Junta de Extremadura a través del Fondo Europeo de Desarrollo Regional. Una manera de hacer Europa. Extremadura. España. (GR18129).

\section{Referencias}

Amado, D., del Villar, F., Leo, F. M., Sánchez-Oliva, D., Sánchez-Miguel, P. A., y García-Calvo, T. (2014). Effect of a multi-dimensional intervention programme on the motivation of physical education students. PLoS One, 9(1), e85275. on the motivation of physical education student
http://dx.doi.org/10.1371/journal.pone.0085275

Arias, B. (2008). Desarrollo de un ejemplo de análisis factorial confirmatorio con LISREL AMOS y SAS. En M. A. Verdugo, M. Crespo, M. Badía, y B. Arias (Eds.), Metodología en la investigación sobre discapacidad. Introducción al uso de las ecuaciones estructurales (pp. 1-42). Salamanca: INICO.

Aron, A., y Aron, E. (2001). Estadística para psicología. Buenos Aires: Pearson Education.

Aspano, M. I., Lobato, S., Leyton, M., Batista, M., y Jiménez, R. (2016). Predicción de la motivación en los estadios de cambio de ejercicio más activos. Retos: Nuevas Tendencias en Educación Física, Deporte y Recreación, 30, 87-91.

Azorín, F., y Sánchez-Crespo, J. L. (1986). Métodos y aplicaciones del muestreo. Madrid: Alianza Editorial.

Batista, M., Jiménez, R., Leyton, M., Lobato, S., y Aspano, M. I. (2016). Adaptation and validation of the Portuguese version of the Healthy Life Styles Questionnaire. International Scientific Researches Journal. PONTE, 72(9), 145-158. http://dx.doi.org/10.21506/j.ponte.2016.9.11

Bentler, P. M. (1995). EQS structural equations program manual. Encino, C.A: Multivariate Software. 
Bollen, K. A. (1989). Structural equations with latent variables. New York: John Wiley y Sons, Inc. http://dx.doi.org/10.1002/9781118619179

Byrne, B. M. (2010). Structural equation modeling with AMOS: Basic concepts, applications, and programming. (2nd ed.). New York:Routledge Taylor \& Francis Group.

Campo-Arias, A., y Oviedo, H. C. (2008). Propiedades psicométricas de una escala: la consistencia interna. Revista de Salud Pública, 10(5), 831-839. http://dx.doi.org/10.1590/S0124-00642008000500015

Carbó-Carreté, M., Guàrdia-Olmos, J., Giné, C., y Schalock, R. L. (2016). A structural equation model of the relationship between physical activity and quality of life. International Journal of Clinical and Health Psychology, 16(2), 147-156. http://dx.doi.org/10.1016/j.ijchp.2015.11.001

Casado-Perez, C., Hernández-Barrera, V., Jiménez-García, R., Fernández-deLas-Peñas, C., Carrasco-Garrido, P., y Palacios-Cena, D. (2015). Physical activity in adult working population: Results from the European National Health Survey for Spain (2009). Atención Primaria, 47(9), 563-572. http://dx.doi.org/10.1016/j.aprim.2015.01.005

Chacón-Cuberos, R., Zurita-Ortega, F., Olmedo-Moreno, E., Padial-Ruz, R., y CastroSánchez, M. (2018). An exploratory model of psychosocial factors and healthy habits in university students of physical education depending on gender. International Journal of Environmental Research and Public Health, 15(11), 2430. http://dx.doi.org/10.3390/ijerph15112430

Chacón-Cuberos, R., Zurita-Ortega, F., Puertas, P., Knox, E., Cofré, C., Viciana, V., y Muros, J. J. (2018). Relationship between healthy habits and perceived motivational climate in sport among university students: A structural equation model. Sustainability, 10(4), 938. http://dx.doi.org/10.3390/su10040938

Charchaoui, I., Cachón, J., Chacón, F., y Castro, R. (2017). Tipos de motivación para participar en las clases de Educación Física en la etapa de Enseñanza Secundaria Obligatoria (ESO). Acción Motriz: Tu Revista Científica Digital, 18(1), 37-46.

Curran, P. J., West, S. G., y Finch, J. F. (1996). The robustness of test statistics to nonnormality and specification error in confirmatory factor analysis. Psychological Methods, 1, 16-29.

Deci, E. L., Olafsen, A. H., y Ryan, R. M. (2017). Self-determination theory in work organizations: The state of a science. Annual Review of Organizational Psychology and Organizational Behavior, 4, 19-43. http://dx.doi.org/10.1146/annurev-orgpsych-032516-113108

Deci, E. L., y Ryan, R. M. (1985). Intrinsic motivation and self-determination in human behavior. New York: Plenum.

Deci, E. L., y Ryan, R. M. (2000). The "what" and "why" of goal pursuits: Human needs and the self-determination of behavior. Psychological Inquiry, 11, 227-268. http://dx.doi.org/10.1207/S15327965PLI1104_01

Deci, E. L., y Ryan, R. M. (2002). Handbook of self-determination research. Rochester, New York: University of Rochester Press.

Egan, C. A., y Webster, C. A. (2018). Using theory to support classroom teachers as physical activity promoters, Journal of Physical Education. Journal of Physical Education, Recreation and Dance, 89(1), 23-29. http://dx.doi.org/10.1080/07303084.2017.1390510

Fernandes, H. M., y Vasconcelos-Raposo, J. (2005). Continuum de auto-determinação: validade para a sua aplicação no contexto desportivo. Estudos de Psicologia, 10(3), 385-395. http://dx.doi.org/10.1590/S1413-294X2005000300007

Fernández-Ozcorta, E. J., Almagro, B. J., y Sáenz-López, P. (2015). Predicción de la intención de seguir siendo físicamente activos en estudiantes universitarios. Cuadernos de Psicología del Deporte, 15(1), 275-284. http://dx.doi.org/10.4321/S1578-84232015000100026

Ferriz, R., González-Cutre, D., Sicilia, Á., y Hagger, M. S. (2016). Predicting healthy and unhealthy behaviors through physical education: A self-determination theorybased longitudinal approach. Scandinavian Journal of Medicine \&Science in Sports, 26(5), 579-592. http://dx.doi.org/10.1111/sms.12470

Franco, E., Coterón, J., Gómez, V., Brito, J., y Martínez, H. A. (2017). Influencia de la motivación y del flow disposicional sobre la intención de realizar actividad físico-deportiva en adolescentes de cuatro países. Retos: Nuevas Tendencias en Educación Física, Deporte y Recreación, 31(1), 46-51.

Franco, E., Coterón, J., Gómez, V., y Laura, A. (2017). Relación entre motivación, actividad física realizada en el tiempo libre y la Intención futura de práctica de actividad física Estudio comparativo entre adolescentes argentinos españoles. SPORT TK-Revista EuroAmericana de Ciencias del Deporte, 6(1), 25-34. http://dx.doi.org/10.6018/280371

Giménez, M. R. L., López, M. P. M., Urda, A. I. M., y Collazos, J.F. R. (2015).Eating habits, health and gender in the elderly. European Journal of Investigation in Health, Psychology and Education, 4(1), 31-40. http://dx.doi.org/10.30552/ejihpe.v4i1.62

González-Cutre, D., Ferriz, R., Beltrán-Carrillo, V. J., Andrés-Fabra, J. A., MonteroCarretero, C., Cervelló, E., y Moreno-Murcia, J. A. (2014). Promotion of autonomy for participation in physical activity: A study based on the trans-contextual model of motivation. Educational Psychology, 34, 367-384. http://dx.doi.org/10.1080/01443410.2013.817325

González-Cutre, D., Sicilia, A., y Moreno, J. A. (2011). Un estudio cuasiexperimental de los efectos del clima motivador tarea en las clases de educación física. Revista de Educación, 356(1), 677-700. https://doi.org/10-4438/1988-592X-RE-2010-356-056

Goudas, M., Biddle, S., y Fox, K. (1994). Perceived locus of causality, goal orientations, and perceived competence in school physical education classes. British Journal of Educational Psychology, 64, 453-463. http://dx.doi.org/10.1111/j.2044-8279.1994.tb01116.x

Hair,J.F., Anderson, R. E., Tatham, R. L., y Black, W. C. (1998).Multivariate data analysis (5th ed). Upper Saddle River, NJ: Prentice Hall.
Hein, V., Müür, M., y Koka, A. (2004). Intention to be physically active after school graduation and its relationship to three types of intrinsic motivation. European Physical Education Review, 10(1), 5-19. http://dx.doi.org/10.1177/1356336X04040618

Hu, L., y Bentler, P. M. (1999). Cutoff criteria for fit indexes in covariance structure analysis: Conventional criteria versus new alternatives. Structural Equation Modeling, 6, 1-55. http://dx.doi.org/10.1080/10705519909540118

Jiménez, R., Cervelló, E., García, T., Santos-Rosa, F. J., y Iglesias, D. (2007). Estudio de las relaciones entre motivación, práctica deportiva extraescolar y hábitos alimenticios y de descanso en estudiantes de Educación Física. International Journal of Clinical and Health Psychology, 7, 385-401.

Jiménez, R., Leyton, M., Batista, M. (2019).Validation of the Intentionality Scale of being physically active in a Portuguese population. Comunicación oral aceptada. En 2019 XV Seminário Internacional de Educação Física, Lazer e Saúde (SIEFLAS) e IV Budo Congress Castelo Branco, Portugal.

Kline, R. B. (2011). Principles and practice of structural equation modeling. Structural equation modeling. New York: Guilford Press., https://doi.org/101038156278a0.

Leyton, M., Batista, M., Lobato, S., Aspano, M. I., y Jiménez, R. (2017). Application of two intervention programs in order to optimize motivation and to improve eating habits in adult and elderly women. Journal of Human Kinetics, 59(1), 131-142. http://dx.doi.org/10.1515/hukin-2017-0153

Leyton, M., García, M., Fuentes, G., y Jiménez, C. (2018). Analysis of motivational variables and healthy lifestyles in sports center practitioners by gender. Retos: Nuevas Tendencias en Educación Física, Deporte y Recreación, 34(1), 166-171.

Mardia, K. V. (1970). Measures of multivariate skewness and kurtosis with applications. Biometrika, 57, 519-530.

McDonald, R. P. (1999). Test theory. A unified treatment. Mahwah, NJ: Lawrence Erlbaum Associates.

McDonough, M. H., y Crocker, P. R. (2007). Testing self-determined motivation as a mediator of the relationship between psychological needs and affective and behavioral outcomes. Journal of Sport and Exercise Psychology, 29(5), 645-663. http://dx.doi.org/10.1123/jsep.29.5.645

Méndez, J. I., y Fernández-Río, J. (2017). Social responsibility, basic psychological needs, intrinsic motivation, and friendship goals in physical education. Retos: Nuevas Tendencias en Educación Física, Deporte y Rrecreación, 32(1), $134-139$.

Montero, I., y León, O. G. (2007). A guide for naming research studies in Psychology, International Journal of Clinical and Health Psychology, 7(3), 847-862.

Moreno-Murcia, J. A., Huéscar, E., y Cervelló, E. (2012). Prediction of adolescents doing physical activity after completing secondary education. The Spanish Journal of Psychology, 15(1), 90-100. http://dx.doi.org/10.5209/rev_SJOP. 2012.v15.n1.37288

Ng, J. Y., Ntoumanis, N., Thøgersen-Ntoumani, C., Deci, E. L., Ryan, R. M., Duda, J. L., y Williams, G. C. (2012). Self-determination theory applied to health contexts: A meta-analysis. Perspectives on Psychological Science, 7(4), 325-340. http://dx.doi.org/10.1177/1745691612447309

Ntoumanis, N., Quested, E, Reeve, J., y Cheon, S. H. (2017). Need supportive communication: implications for motivation in sport, exercise, and physical activity. Persuasion and communication in sport, exercise and physical activity. Abingdon, UK: Routledge.

Nunnally, J. C. (1978). Psychometric theory (2nd ed.). New York: McGraw-Hill.

Nunnally, J. C., y Bernstein, I. H. (1994). Psychometric theory (3rd ed.). New York: McGraw-Hill.

Núñez, J. L., y León, J. (2016). The mediating effect of intrinsic motivation to lear non the relationship between student's autonomy support and viality and deep learning. The Spanish Journal of Psychology, 19(42), 1-6. http://dx.doi.org/10.1017/sjp.2016.43

Pires, A., Cid, L., Borrego, C., Alves, J., y Silva, C. (2010). Validação preliminar de um questionário para avaliar as necessidades psicológicas básicas em Educação Física. Motricidade, 6(1), 33-51. http://dx.doi.org/10.6063/motricidade.6(1).157

$R$ Core-Team (2014). R: A language and environment for statistical computing [Internet]. 2014 [acceso 20 Sep 2018]. Disponible en: http://www.R-project.org.

Revelle, W. (2014). Psych: Procedures for psychological, psychometric and personality research. Illinois: Evanston.

Revelle, W., y Zinbarg, R. E. (2009). Coefficients alpha. omega, and the Gbl: Comments on sijtsma. Psychometrika., 74(1), 145-154. http://dx.doi.org/10.1007/s11336-008-9102-z

Ryan, R. M., y Deci, E. L. (2000). Self-determination theory and the facilitation of intrinsic motivation, social development, and well-being. The American Psychologist, 55, 68-78. http://dx.doi.org/10.1037/0003-066X.55.1.68

Ryan, R. M., y Deci, E. L. (2017). Self-determination theory: Basic psychological needs in motivation development and wellness. New York: Guilford Publishing.

Saavedra, J. M., García-Hermoso, A., Escalante, Y., y Domínguez, A. M. (2014) Self-determined motivation, physical exercise and diet in obese children: A three-year follow-up study. International Journal of Clinical and Health Psychology, 14(3), 195-201. http://dx.doi.org/10.1016/j.ijchp.2013.12.001

Sanchez, B., Byra, M., y Wallhead, T. L. (2012). Students' perceptions of the command, practice, and inclusion styles of teaching. Physical Education \& Sport Pedagogy, 17(3), 317-330. http://dx.doi.org/10.1080/17408989.2012.690864

Sánchez-Oliva, D., Leo, F. M., Sánchez-Miguel, P. A., Amado, D., y García-Calvo, T. (2013). Desarrollo de un modelo causal para explicar los comportamientos positivos en las clases de educación física. Acción Motriz, 10, 48-58.

Sánchez-Oliva, D., Pulido-González, J. J., Leo, F. M., González-Ponce, I., y García-Calvo, T. (2017). Effects of an intervention with teachers in the physical education context: A self-determination theory approach. PLoS One, 12(12), e0189986. http://dx.doi.org/10.1371/journal.pone.0189986 
Standage, M., Duda, J. L., y Ntoumanis, N. (2005). A test of self-determination theory in school physical education. British Journal of Educational Psychology, 75(3) 411-433. http://dx.doi.org/10.1348/000709904X22359

Standage, M., Duda, J. L., y Ntoumanis, N. (2006). Students' motivational processes and their relationship to teacher ratings in school physical education: A selfdetermination theory approach. Research Quarterly for Exercise and Sport, 77(1), 100-110. http://dx.doi.org/10.1080/02701367.2006.10599336

Taylor, I. M., y Ntoumanis, N. (2007). Teacher motivational strategies and student self-determination in physical education. Journal of Education Psychology, 99(2), 747-760. http://dx.doi.org/10.1037/0022-0663.99.4.747

Taylor, I. M., Ntoumanis, N., Standage, M., y Spray, C. M. (2010). Motivational predictors of physical education students' effort, exercise intentions, and leisure-time tors of physical education students' effort, exercise intentions, and leisure-time
physical activity: A multilevel linear growth analysis.Journal of Sport and Exercise physical activity: A multilevel linear growth analysis. Journal of Spor
Psychology, 32(1), 99-120. http://dx.doi.org/10.1123/jsep.32.1.99

Thompson, D., Cantu, D., Bhatt, R., Baranowski, T., Rodgers, W., Jago, R., Buday, R. (2014). Texting to increase physical activity among teenagers (TXT Me!): Rationale, design, and methods proposal. JMIR Research Protocols, 3(1), 14 http://dx.doi.org/10.2196/resprot.3074

Usán, P., Salavera, C., Mejías, J. J., y Murillo, V. (2018). Orientación motivacional y percepción de promoción del bienestar en profesorado de educación física hacia sus alumnos. Retos: Nuevas Tendencias en Educación Física, Deporte y Recreación, $33,46-49$.

Vallerand, R. J. (1997). Toward a hierarchical model of intrinsic and extrinsic motivation. En M. P. Zanna (Ed.), Advances in experimental social psychology (29) (pp. 271-360). New York, San Diego: Academic Press.
Vallerand, R. J. (2001). A hierarchical model of intrinsic and extrinsic motivation in sport and exercice. En G. C. Roberts (Ed.), Advances in motivation in sport and exercise (pp. 263-319). Champaign, IL: Human Kinetic.

Vansteenkiste, M., Lens, W., y Deci, E. L. (2006). Intrinsic versus extrinsic goal contents in self-determination theory: Another look at the
quality of academic motivation. Educational Psychology, 41(1), 19-31. quality of academic motivation. Educational

Vansteenkiste, M., Niemiec, C., y Soenens, B. (2010). The development of the five mini-theories of self-determination theory: An historical overview, emerging trends and future directions. En T. Urdan y S. Karabenick (Eds.), Advances in motivation and achievement.The decade ahead (16) (pp. 105-166). Bingley, UK: Emerald. http://dx.doi.org/10.1108/S0749-7423(2010)000016A007

Vlachopoulos, S. P., y Michailidou, S. (2006). Development and initial validation of a measure of autonomy, competence, and relatedness: The Basic Psychological Needs in Exercise Scale. Measurement in Physical Education and Exercise Science, 10, 179-201. http://dx.doi.org/10.1207/s15327841mpee1003_4

Wold, B. (1995). Health behavior in school children: A WHO cross-national survey. Resource package questions. pp. 1993-1994. Norway: University of Bergen.

Yilmaz, A., Esenturk, O. K., Demir, G. T., y Ilhan, E. L. (2017). Metaphoric perception of gifted students about physical education course and physical education teachers. Journal of Education and Learning, 6(2), 220-234. http://dx.doi.org/10.5539/jel.v6n2p220 
